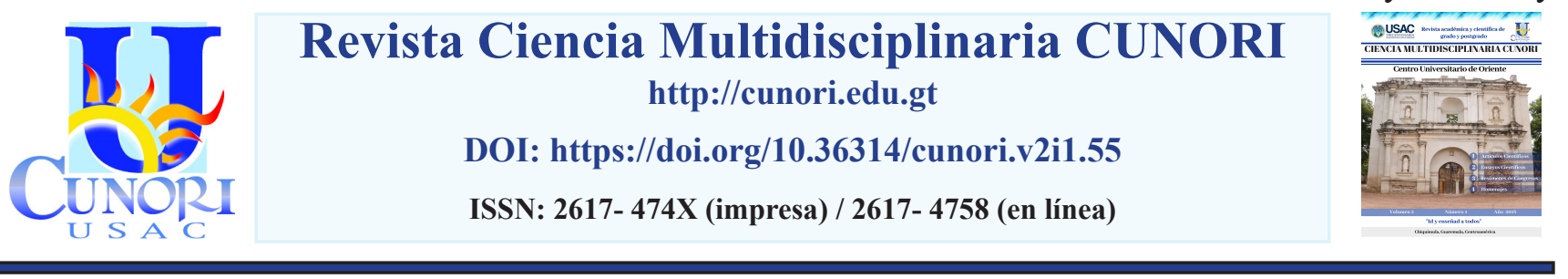

Como citar el ensayo

Esquivel, M. \& Villela, C. (2018). La reingeniería para mejorar el desempeño del recurso humano. Revista Ciencia Multidisciplinaria CUNORI, 2(1), 71-78. https://doi.org/10.36314/cunori.v2i1.55

\title{
La reingeniería para mejorar el desempeño del recurso humano
}

\section{Reengineering to improve the performance of human resources}

\author{
Margareth Esquivel* \& Claudia Villela \\ Maestría en Administración de Recursos Humanos , Universidad Mariano Gálvez de Guatemala \\ Recibido: 14 de julio de 2018 Aceptado: 14 de agosto de 2018 \\ Disponible en internet el 17 de agosto de 2018
}

\author{
*Autor para correspondencia. \\ Resumen \\ Correo electrónico: marand89@hotmail.com
}

Dealizar reingeniería es de gran importancia, más aún cuando el enfoque está puesto en el desempeño del recurso humano, pues desde siempre ha sido la clave para el desarrollo de la organización. La investigación que se presenta es del tipo bibliográfica descriptiva, la cual incluye argumentos y diversos puntos de vista de expertos, que desde la década de los 90', han dado a conocer sus aportes en cuanto a la aplicación de la reingeniería. Por ejemplo, Delgado, presenta un concepto muy claro de reingeniería, que consiste en empezar desde cero, Chiavenato, quien es uno de los autores más conocidos, menciona la innovación a través de la reingeniería para mantener a las organizaciones a la vanguardia, Juárez por otro lado considera que no se deben olvidar considerar al momento de hacer reingeniería, los esquemas actuales de alta productividad los cuales deberían ser acordes con los salarios. Las jornadas de trabajo son demasiado amplias y reducen el tiempo para la vida personal, ya que la mayoría acepta jornadas extraordinarias buscando obtener ingresos adicionales. Tomando en consideración las opiniones de algunos expertos con respecto a la reingeniería y los cambios que a través de ella se puedan aplicar en el desempeño de los colaboradores.

Palabras clave: reingeniería, desempeño de recursos humanos, cambio

Abstract

D eengineering is of great importance, especially when the focus is on the performance of human resources, since it has always Reen the key to the development of the organization. The research that presents the type of descriptive bibliography, which includes arguments and several points of view of experts, who since the decade of the 90 ', have made known their arguments regarding the application of reengineering. For example, Delgado, presents a very clear concept of reengineering, which consists of starting from scratch, Chiavenato, who is one of the best-known authors, innovation through reengineering to keep organizations at the forefront, Juarez on the other hand considers that it is not necessary to stop considering the moment to do the reengineering, the present schemes of the high productivity of which they will be in agreement with the wages. The work days are too numerous and reduce the time for personal life, since most accept extraordinary days. Taking into account the opinions of some experts regarding the reengineering, the changes that through it can be applied in the performance of the collaborators.

Keywords: reengineering, performance of human resources, change 


\section{Introducción}

La reingeniería aporta a las organizaciones grandes oportunidades de cambio, por lo cual es necesario conocer a fondo este tema, con el fin de poder determinar qué tan importante es realizar un proceso de este tipo para mejorar el desempeño del recurso humano dentro de las organizaciones. Se afirma erróneamente, que Michael Hammer es el pionero de la reingeniería, sin embargo, el mismo autor lo rechaza, al afirmar que "las empresas venían poniendo en práctica la reingeniería con anterioridad" (Hammer y Champy, 1994).

El objetivo del ensayo es dar a conocer las opiniones principales de algunos autores con experiencia en el tema, pero a la vez conocer más de reingeniería y el beneficio que representa en el desempeño laboral. No la inventaron, sino que le dieron nombre y divigulgación. De cualquier forma la reingeniería tiene sus bases en otros autores, al formular definiciones que en gran medida conservan los postulados originales. Si la reingeniería en un cambio radical al proporcionar un enfoque global en la reconstrucción de procesos de una organización, sin embargo no debe confundirse con Calidad total, Downsizing, Benchmarking.La participación del departamento de personal ayuda a identificar problemas mientras se cuente con el tiempo para solucionarlos, además, aporta información relacionada con los colaboradores. En este sentido, los nuevos procesos deben ser compatibles con las políticas corporativas del personal. Se determina que, para poder realizar este proceso debe de existir compromiso de toda la organización y también cero resistencias al cambio.

\section{¿Qué es reingeniería en el desempeño de los recursos humanos?}

"Durante el transcurso de su desarrollo la administración moderna, ha logrado descomponer actividades complejas en pasos pequeños y repetibles" (Hamel \& Breen, 2008). La reingeniería es el proceso aplicado a la administración, mediante el cual se pretende cambiar todo, para mejorar los procesos y el desempeño de los colaboradores, pero es necesario tener como enfoque el recurso humano, para que sea el punto de partida para mejorar en todas áreas. En lo que respecta al mejoramiento radical, durante las últimas dos décadas se ha difundido un procedimiento conocido como reingeniería, cuyo enfoque consiste en reinventar los procesos a partir de cero, como lo afirma (Delgado, 2011), es un proceso que transforma totalmente los procesos de una empresa, en este caso el desempeño del recurso humano es básico para mejorar el rendimiento de los colaboradores y desde luego la empresa alcanzará metas.

Según (Chiavenato, 2009) las grandes organizaciones dejaron a un lado el organigrama tradicional y empezaron a innovar en términos de estructura organizacional y de organización del trabajo. No lo hicieron por la simple vanidad de ostentar una estructura moderna, sino por la imperiosa necesidad de volverse más ágiles, flexibles, innovadores y rápidos, con la idea de terminar con las antiguas estructuras organizacionales que limitaban a las personas en su trabajo, por lo tanto la reingeniería si se aplica a una organización tiene que tener mucho énfasis en la gestión del recurso humano, no solo por ser el recurso más valiosos de la empresa, sino también porque si reestructuramos dichos sistemas las personas tendrían más confianza y se sentirían más identificadas con la empresa, estos los conviertirá en colaboradores más activos y eficaces para cualquier organización. 
Según (Gómez, L. et.al. 2008) la reingeniería analiza la forma en que la empresa lleva a cabo sus actividades prestando una atención minuciosa a los procesos centrales implicados en la producción de sus productos o en la entrega de sus servicios al consumidor. Con el aprovechamiento de la tecnología informática y las distintas maneras de organizar al recurso humano, la empresa puede ser capaz de volver a inventarse a sí misma.

Si se pretender llevar a cabo en una empresa es necesario empezar, aprovechando el recurso humano con el que se cuenta y la capacidad que puedan desarrollar para adaptarse a las tendencias, para ello no solo se tiene que capacitar a las personas, sino que también contar con la tecnología mas avanzada y adaptarse a los procesos que crearán un vínculo con el desempeño de las personas en la organización, con el propósito de generar más beneficios utilizando menos recursos. Los esquemas actuales de alta productividad deberían ir acordes con los salarios dignos. Las jornadas de trabajo son demasiado amplias y reducen el tiempo para la vida personal, ya que la mayoría acepta jornadas extraordinarias buscando obtener ingresos adicionales. Las organizaciones que han "simplificado procesos", mediante reingeniería o técnicas similares, no han disminuido el trabajo, sino han reducido su la planilla de personal. La consecuencia inmediata de esto es que los empleados que continúan en la empresa deben efectuar las labores que otros hacían (Juárez, 2013:53). La productividad de un grupo de personas va muy ligado a los salarios, porque de ellos depende las actividades que realizan en su vida personal y familiar, por lo que se puede notar cuando las personas trabajan aprovechando su potencial, realizan el trabajo en menor tiempo y de mejor manera, para lo cual no se necesitan tantas personas o tiempo, solo se necesita darles a las personas los beneficios y la motivación que merecen.

Tiene que estar consciente de que incluso los cambios pequeños en las organizaciones suelen ser difíciles de realizar. Si los resultados indican grandes cambios en la política organizacional, el analista puede esperar resistencia. Al analizar los resultados, debería comprobar quién debe cambiar y cuánto, si las personas que lo harán estarán mejor o peor, y quién tendrá el poder de dirigir el cambio (Render, 2012). Si el cambio es pequeño es difícil de asimilar. Un proceso tan complejo como la reingeniería puede provocar un efecto negativo en los colaboradores, haciendo que no se sientan identificados o tomados en cuenta en las decisiones importantes, pero es necesario cambiar y hacer ver a los colaboradores que ellos se beneficiarán también. Desde el momento en que se decide realizar una reingeniería el personal debe ser consciente de que puede haber recortes, y los que queden serán capacitados y adaptados a los nuevos procesos de la empresa, quizás por tener conocimiento al respecto de esta información los empleados se sentirán inseguros.

Según (Marte, 2016) en todas las organizaciones cada posición tiene definido un conjunto de requerimientos los cuales definen que perfil debe tener el recurso humano, para poder ocuparla, de la misma manera, el personal, que ocupa una posición dentro de la empresa debe ser evaluado con relación a lo que requiere, de acuerdo con los objetivos empresariales. La necesidad de realizar una reingeniería es latente en muchas organizaciones que perdieron el enfoque y más aún cuando muchos no tienen ni el control de sus áreas de trabajo, muchas veces las ordenes llegan de diferentes personas y esto hace que los colaboradores se confundan y se les complique el poder alcanzar sus objetivos. 
Según (García, 2014) la importancia de los recursos humanos para el éxito del proyecto de reingeniería hace que esta área deba recibir atención desde el inicio. La participación del departamento de personal puede ayudar a identificar problemas mientras se cuenta con el tiempo para solucionarlos, además, aporta información relacionada con el personal, obviamente resulta deseable que los nuevos procesos sean compatibles con las políticas corporativas del personal. La estrategia de reingeniería permite analizar los procesos desde antes de su aplicación para poder anticiparse a los problemas con los que la empresa se encontró en algún momento.

Reingeniería según (Chamber, 2012) es un enfoque gerencial moderno que permite revalorizar el capital humano en la organización con el propósito de lograr una fuerza de trabajo más dinámica, eficiente y competitiva. Esta estrategia también ayuda a que los colaboradores tengan mayor importancia y participación en la organización, pues la reingeniería tendría ese enfoque y brindaría al recurso humano, más herramientas para aprovechar sus capacidades y habilidades, todo esto conllevará el desempeño de las personas a un nivel altamente competitivo.

Según (Pulido, 2010) todo el mundo, principalmente la gente que tiene el mando, es responsable de mejorar el desempeño de sus procesos. La alta dirección encabeza el esfuerzo para generar visiones compartidas, alinear los esfuerzos dentro de la compañía, eliminar barreras organizacionales, propiciar el aprendizaje organizacional y facultar y potenciar el trabajo y la creatividad del talento humano de la organización. El aprendizaje es el mejor aliado para que la estrategia de reingeniería funcione, puesto que, si el enfoque es mejorar el desempeño del recurso humano, es necesario no solo hacer una nueva estructura organizacional, sino que también es necesario darles más valor a las personas y hacer que sus esfuerzos sean tomados más en cuenta, para que se sientan como parte vital de la organización y por ende se preparen más para seguir creciendo en la empresa. Los planes estratégicos cuando realizan cambios radicales en la empresa, tiene que ir encaminados hacia el desempeño de todas las áreas, con ello se busca que todos persigan los mismos objetivos, si esto se logra, los procesos serán mucho más fáciles de entender y también de realizar para los colaboradores.

Para (Evans \& Lindsay, 2008) el mejoramiento y el aprendizaje continuo deben ser parte regular del trabajo diario; deben ser practicados a nivel personal, de unidad en el trabajo, impulsados por las oportunidades para incidir en un cambio significativo y centrarse en compartir en la organización. El mejoramiento continuo se logra con la práctica, no solamente de forma individual, sino también en equipo, esto ayuda a que todos se ayuden y motiven para mejorar constantemente, además de ser más eficientes para la empresa. Por esta razón al realizar cualquier estrategia que tenga que ver con cambios, siempre el recurso humano tiene que ser prioritario.

\section{Las etapas para hacer reingeniería según (Mallar, 2010)}

\section{Etapa 1 - Información, formación y participación}

Cuando se trata de adoptar una nueva metodología y cambiar la forma de pensar y de trabajar de las personas, es esencial la información y también la formación que se les brinde. Por ello, la implementación de la gestión en base a los procesos debe realizarse de la forma más participativa posible. Se debe informar al personal sobre cuáles son los objetivos del proceso, sus etapas, los resultados esperados, la colaboración requerida, etc. Organizando talleres que permitan formar al personal. 


\section{Etapa 2 - Identificación de los procesos y definición de las fronteras de cada uno.}

El nombre con que se identifique a cada proceso debe representar claramente lo que se hace en él. Todas las actividades que se llevan a cabo en la organización, deben estar incluidas en alguno de los procesos listados. En caso contrario no son relevantes o importantes por lo cual se pueden descartar. Aunque el número de procesos depende del tipo de empresa, si se identifican pocos procesos o por el contrario demasiados, se aumentan las dificultades de gestión posterior. Con los procesos identificados, cada grupo de trabajo definirá el mapa de procesos que le corresponde, tratando de verificar cuáles son los procesos importantes que se realizarán. Se tendrán que analizar también, los límites del proceso identificando las entradas y salidas, reconociendo a los proveedores y a los clientes del proceso, así como aquellos otros procesos con que tiene alguna relación.

\section{Etapa 3 - Selección de los procesos clave}

Una vez establecido el listado de todos los procesos, deben diferenciarse los procesos relevantes y los procesos clave. Una de las características principales que normalmente tienen los procesos relevantes es que son interfuncionales, pudiendo cruzar vertical y horizontalmente la organización. En tanto los procesos clave son aquellos procesos que forman parte de los procesos relevantes y que inciden de manera significativa en los objetivos estratégicos, siendo críticos para el éxito del negocio.

\section{Etapa 4 - Nombrar al responsable del proceso}

Cuando han sido seleccionados los procesos relevantes y claves, se debe nombrar un responsable o propietario, para cada uno de ellos (el dueño del proceso). A partir de ese momento el responsable del proceso contará con autonomía de actuación y con la responsabilidad de dar respuesta a los objetivos estratégicos.

\section{Etapa 5 - Revisión y análisis de los procesos y detección de los problemas}

En esta instancia hay que analizar cada proceso, partiendo de los más importantes. Elegido el proceso, hay que verificar de qué manera éste da respuesta a los objetivos estratégicos, y si no es así, habrá que abordar el diseño o rediseño del proceso.

\section{Etapa 6 - Corrección de los problemas}

A partir de los resultados de la etapa anterior, donde han quedado definidos los problemas que presenta el proceso y que tienen mayor incidencia sobre los objetivos estratégicos de la organización, se considerarán las posibilidades reales de solución a los problemas de forma viable para la organización, a corto plazo, analizándose las posibles acciones a seguir para solucionar los que mayor efecto tienen sobre el desempeño del proceso, considerando su factibilidad de aplicación y el impacto integral sobre todo el sistema. 


\section{Etapa 7 - Establecimiento de indicadores}

Los procesos deben ser evaluados periódicamente ya que partiendo de las evaluaciones que se realicen, se pueden determinar los puntos débiles y de esta forma establecer una estrategia completa encaminada a mejorar su funcionamiento. La utilización de indicadores es fundamental para poder interpretar lo que está ocurriendo, y tomar medidas cuando las variables se salen de los límites establecidos o márgenes de tolerancia. Servirán también para definir las necesidades de introducir cambios y poder evaluar sus consecuencias, como así para planificar actividades destinadas a dar respuesta a nuevas necesidades. Se plantea por lo tanto la necesidad de definir indicadores dando respuesta a las siguientes preguntas: ¿Qué debemos medir?, ¿Dónde es conveniente medir?, ¿Cuándo hay que medir? ¿En qué momento o con qué frecuencia?, ¿Quién debe medir?, ¿Cómo se debe medir?, ¿Cómo se van a difundir los resultados?, ¿Quién y con qué frecuencia va a revisar y/o auditar el sistema de obtención de datos?

\section{El desempeño laboral para el cambio según (Ortega, 2015)}

La capacidad integral que tiene una persona para desempeñarse eficazmente en situaciones específicas de trabajo. Son aquellos conocimientos, destrezas y aptitudes necesarias para ejercer una profesión, puede resolver los problemas profesionales de forma autónoma y flexible. Estar capacitado para colaborar en su entorno profesional y en la organización del trabajo, es una construcción social de aprendizajes significativos y útiles para el desempeño productivo en una situación real de trabajo que se obtiene, no sólo a través de la instrucción, sino también -y en gran medida, mediante el aprendizaje por experiencia en situaciones concretas de trabajo.

Una construcción, a partir de una combinación de recursos como los conocimientos, saber hacer, cualidades o aptitudes, y recursos del ambiente, las relaciones, documentos, informaciones y otros, que son movilizados para lograr un buen desempeño. Las técnicas, las habilidades, los conocimientos y las características que distinguen a un colaborados destacado, por su rendimiento, sobre un normal dentro de una misma función o categoría laboral.

El análisis de competencias tiene como objeto Identificar los conocimientos, las destrezas, las habilidades y los comportamientos estimulantes, que los empleados deben demostrar para que la organización alcance sus metas y objetivos. El concepto de competencia surge de la necesidad de valorar no sólo el conjunto de los conocimientos apropiados (saber) y las habilidades y destrezas (saber hacer) desarrolladas por una persona, sino de apreciar su capacidad de emplearlas para responder a situaciones, resolver problemas y desenvolverse en el mundo. Igualmente, implica una mirada a las condiciones del individuo y disposiciones con las que actúa, es decir, al componente actitudinal y valorativo (saber ser) que incide sobre los resultados de la acción.

\section{Conclusión}

Después de más de veinte años, desde que surgió la Reingeniería, las organizaciones, no han dejado de aplicarla, especialmente en el departamento de recursos humanos, en los procesos de mejoramiento del desempeño de los colaboradores. Hacer sentir a los colaboradores como parte esencial de la organización es de gran ayuda motivacional y mejora el rendimiento de cualquier área, por lo cual es realmente necesario que tome partida cada área, definitivamente la efectiva relación entre las áreas de la 
organización en los procesos de reingeniería, es lo que lo hará exitoso. El mismo debe estar enfocado al aprovechamiento de las habilidades y el talento de las personas, a la vez que le da la oportunidad a algunos colaboradores que normalmente son puestos a un lado, la oportunidad de ser líderes y proponer cambios que beneficien a la empresa, gestionando de mejor manera sus habilidades y recursos, aprovechando algunos métodos provenientes de la forma en la que otras empresas aplicaron la estrategia de reingeniería. Las etapas planteadas permiten desarrollar una adecuado proceso de reingeniería, basado en normas ISO 9000, por medio de la identificación dentro de la estructura de procesos establecidos, para alcanzar los objetivos. Demostrando el recurso humano es un elemento de utilidad para una gestión eficiente. Realizar la reingeniería en una empresa realmente es arriesgado, pero si se proveen todos los posibles acontecimientos, será de gran ayuda para mantener a la organización a la vanguardia, además de mantener a los colaboradores en constante capacitación y aprendizaje.

\section{Referencias bibliográficas}

Chamber, A. (2012). MBS Consulting. Obtenido de MBS Consulting: https://www.mbsperu.com/ publicaciones/presentaciones-en-ppt/consultoria-rrhh/reingenieria-de-recursos-humanos

Chiavenato, I. (2009). Gestión del Talento Humano. En I. Chiavenato, Gestión del Talento Humano (pág. 404). Mexico, D.F: McGraw-Hill.

Delgado, H. (2011). Desarrollo de una Cultura de Calidad. En H. C. Delgado, Desarrollo de una Cultura de Calidad (págs. 163-164). Mexico, D.F.: The McGraw-Hill.

Evans, J. \& Lindsay, W. (2008). Adminsitración y Control de la Calidad. En J. R. Evans, \& W. M. Lindsay, Adminsitración y Control de la Calidad (pág. 23). México, D.F.: Cenagage Learning.

García, E. (2014). Conocimientosweb.net. Obtenido de Conocimientosweb.net : https://www.conocimientosweb.net/portal/article794.html Consultado el 11 de julio 2018.

Gómez, L. et.al. (2008). Gestión de Recursos Humanos. En L. R. Gómez, D. B. Balkin, \& R. L. Cardy, Gestión de Recursos Humanos (pág. 66). Madrid, España: Pearson Educació, S.A.,.

Hamel, G., \& Breen, B. (2008). El Futuro de la Administracion. En G. Hamel, \& B. Breen, El Futoro de la Administracion (pág. 10). Bogotá, Colombia: Grupo Editorial Norma.

Hammer, M. Champy, J. (1994). Reengineering the Corporation: A Manifesto for Business Revolution. New York: Ed. Harperbusiness.

Juárez, R. (2013). Administración de la Compensación. En R. A. Juárez, Administración de la Compensación (pág. 53). Naucalpan de Juárez, Estado de México: Pearson Educación.

Mallar, M. (2010). La Gestión por Procesos: un enfoque de gestión eficiente. Visión de futuro, 13(1)

Marte, F. (2016). Blogs EQI. Obtenido de Blogs EQI: http://www.eoi.es/blogs/mintecon/2015/04/22/ la-evaluacion-de-desempeno-rrhh/ Consultado el 11 de julio 2018.

Ortega, M. (2015). Tesis La resistencia al cambio y influencia en el desempeño laboral. Perú. Universidad Nacional Jorge Basadre Grohmann.

Pulido, H. G. (2010). Calidad Total y Productividad . En H. G. Pulido, Calidad Total y Productividad (pág. 16). México, D.F.: Th e McGraw-Hill. 
Render, R. (2012). Métodos Cuantitativos para los Negocios. En R. M. Barry Render, Métodos Cuantitativos para los Negocios (pág. 16). Naucalpan de Juárez, Estado de México: Pearson Educación de México, S.A. de C.V.

\section{Sobre la autora}

\section{Margaret Esquivel Andrade}

Licenciada en Administración de Empresas con estudios de Maestría en Administración de Recursos Humanos, egresada de Universidad Mariano Gálvez de Guatemala, se desempeña como Gerente en Corporación Internacional Multi inversiones divisiones de Restaurantes de Centro América.

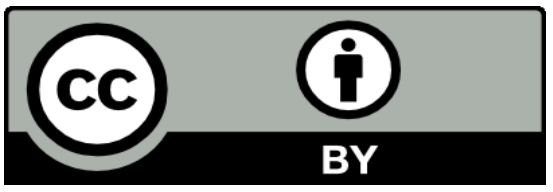

Este texto está protegido por una licencia CreativeCommons 4.0.

Usted es libre para compartir, copiar y redistribuir el material en cualquier medio o formato y adaptar el documento, remezclar, transformar y crear a partir del material para cualquier propósito, incluso comercialmente, siempre que cumpla la condición de atribución: usted debe reconocer el crédito de una obra de manera adecuada, proporcionar un enlace a la licencia, e indicar si se han realizado cambios. Puede hacerlo en cualquier forma razonable, pero no de forma tal que sugiera que tiene el apoyo del licenciante o lo recibe por el uso que hace. 